\title{
Análisis documental de los FIBRAS y su operacionalización en México (Mexican REIT analysis and the Mexican operationalization)
}

\author{
Alan Quiroga Gamboa • \\ Martha del Pilar Rodríguez Garcia * \\ Alma Berenice Méndez Sáenz * \\ Héctor Horacio Garza Sánchez *
}

\begin{abstract}
In this documental research work they are presented as the origin of fideicomisos de infraestructura y bienes raíces or FIBRA, the development of this type of investment instruments in the world, as well as the legal and fiscal structure of the FIBRA in Mexico. For this, the Real Estate Investment Trust or REITs are presented, which are an investment mechanism that finance large real estate not only in America, but throughout the world. This allows the public money market to participate in these investments, although in their history they have presented different risks and yields due to the legal framework of their countries of origin. In Mexico, the legal structure of the FIBRA is very robust and similar to that of the USA. One of the engines of these instruments is the tax exemption they offer; for this reason, the case of Spain has not awakened, it still does not motivate the interest of the market insofar as it does not offer these tax benefits. There is a great appetite all over the world for these instruments. The big question will be to what extent are viable options to use them as a diversification mechanism in order to decrease risk or increase performance.
\end{abstract}

Key words: alternatives on investment, financial performance, financial portfolios, Mexican REIT, MT

JEL: G400, G110 \& G150.

\footnotetext{
- Universidad Autónoma de Nuevo León, Facultad de Contaduría Pública y Administración, Av. Universidad S/N, San Nicolás de los Garza , N.L., México, C.P. 66455, alanquirogagamboa@gmail.com

* Universidad Autónoma de Nuevo León, Facultad de Contaduría Pública y Administración, Av. Universidad S/N, San Nicolás de los Garza, N.L., México, C.P. 66455, marthadelpilar2000@yahoo.com

* Universidad Autónoma de Nuevo León, Facultad de Ingeniería Mecánica y Eléctrica, Av. Universidad S/N, San Nicolás de los Garza, N.L., México, C.P. 66455, beremendez@gmail.com

- Universidad Autónoma de Nuevo León, Facultad de Contaduría Pública y Administración, Av. Universidad S/N, San Nicolás de los Garza, N.L., México, C.P. 66455, hfacpya@hotmail.com
} 
Resumen. En este trabajo de investigación documental se presentan como el origen de los fideicomisos de infraestructura y bienes raíces o FIBRA, el desarrollo de este tipo de instrumentos de inversión en el mundo, además de la estructura legal y fiscal de los FIBRA en México. Para ello se presentan los Real Estate Investment Trust o REITs que son un mecanismo de inversión que financian grandes propiedades inmobiliarias no sólo en América, sino en todo el mundo. Esto permite que el mercado de dinero público participe en estas inversiones, aunque en su historia han presentado diferentes riesgos y rendimientos debido al marco legal de sus países de origen. En México, la estructura legal de los FIBRA es muy robusta y parecida a la de EUA. Uno de los motores de estos instrumentos es la exención fiscal que ofrecen; por ello, el caso de España no han funcionado estos instrumentos y siguen sin motivar el interés del mercado en la medida en que no ofrece estos beneficios fiscales. Existe un gran apetito en todo el mundo por estos instrumentos. La gran pregunta será hasta qué punto son opciones viables para usarlos como mecanismo de diversificación a fin de disminuir el riesgo o aumentar el rendimiento.

Palabras clave: alternativas de inversión, desempeño financiero, FIBRA, portafolio financiero, TPM

\section{Introducción}

En este trabajo de investigación documental se presentan como el origen de los FIBRA, el desarrollo de este tipo de instrumentos de inversión en el mundo, además de la estructura legal y fiscal de los fideicomisos de infraestructura y bienes raíces FIBRA en México con algunas implicaciones en el contexto mundial. Para ello se presentan fideicomisos de infraestructura y bienes raíces los Real Estate Investment Trust o REITs.

Los fondos de pensiones, las administradoras de fondos para el retiro y muchas instituciones financieras consideran a los REITs/FIBRA como un mecanismo de inversión que buscan agregar a sus portafolios. Dicho interés deriva desde que, en 2016 en México ya que alrededor de 36.6\% del dinero público ya estaba invertido en estos instrumentos (SHCP, 2015).

Los fondos de pensiones, las administradoras de fondos para el retiro y muchas instituciones financieras consideran a los REITs/FIBRA como un mecanismo de inversión que buscan agregar a sus portafolios. Cabe aquí preguntarse si lo anterior es una buena idea o representa un riesgo para los inversionistas, pues en la historia internacional de estos tipos de productos han tenido resultados mixtos. 
El aumento de la difusión de la información financiera permite que las acciones de los REIT se vendan rápidamente a precios muy cercanos al valor real del mercado. Gracias al bajo costo de transacción aunado a las acciones, los grandes inversionistas institucionales pueden implementar técnicas de asignación de activos para incrementar o disminuir la exposición a varios sectores del mercado de bienes raíces, mientras mantienen un portafolio sólido (Souza, 2014).

El propósito general de esta investigación es conocer las ventajas y riesgos a los que se enfrentan los inversionistas con el uso de los REITs/FIBRAS en un contexto mundial y en particular el caso mexican. Para ello, el artículo se divide en cuatro apartados, en primer lugar se presentan los antecedentes de la evolución de los REITs en el mundo. En segundo lugar, se presenta un marco teórico sobre las ventajas y riesgos de los REITs en el mundo, en tercer lugar la metodología empleada a través de un análisis de simitudes y diferencias entre los mercados y por último las conclusiones.

\section{Antecedentes}

Los REIT fueron creados en la década de 1960 en EUA dada la necesidad de los bancos de inversión y del gobierno para ofrecer un producto de inversión de alto valor orientado a los fondos de pensiones y otros inversionistas que consideraban a los bienes raíces como una opción menos riesgosa que las acciones o los bonos. Los REIT fueron diseñados para reducir los riesgos de inversión y ampliar la transparencia de los mercados de valores (Graff, 2001).

Los REIT tuvieron un periodo de modesta popularidad durante sus primeros años, aunque no lograron convertirse en una fuente importante de capital para la industria de los bienes raíces (Decker, 1998). Por otra parte, debido a que algunos gerentes de REIT hicieron mal uso de la financiación de la deuda, muchos fideicomisos se disolvieron y el interés de los inversionistas se desvaneció rápidamente durante los mercados bajistas de 1970 (Graff, 2001). En ese momento, los REIT que se mantuvieron en operación se comportaron como acciones y no como propiedades inmobiliarias privadas (Gyourko y Keim, 1992).

Durante las primeras dos décadas de su existencia los REIT no pudieron proporcionar buenos rendimientos inmobiliarios combinados con la liquidez del mercado de valores. Una razón adicional para el lento 
crecimiento de esta industria fueron las restricciones gubernamentales, que sólo permitían invertir en los pasivos de los REIT y prohibían manejar y administrar sus propios inmuebles. Lo anterior impidió que estos fideicomisos evolucionaran y, por el contrario, experimentaran conflictos de agencia entre las compañías operativas y los administradores de los REIT.

Las exenciones fiscales suponían que los REIT podrían adaptarse para permitir inversiones en grandes portafolios diversificados en bienes raíces, combinado con la liquidez del mercado de valores. Sin embargo, las severas restricciones de los REIT se enfrentaron con las limitaciones operativas para administrar estos inmuebles, lo cual impidió, de manera significativa, su crecimiento.

En 1974, el sector de los fondos de pensiones experimentó un cambio importante con la promulgación de la Ley de Seguridad del Empleado de Ingresos de Jubilación (ERISA), que presionó a los administradores de planes de pensiones a diversificar sus inversiones de conformidad con la Teoría de Portafolio Moderno (TPM). Como resultado, los administradores tenían la responsabilidad personal por la falta de diversificación cuando obtuvieran bajos rendimientos. Cabe aclarar que los planes de pensiones tenían la finalidad de eliminar las restricciones legales en las inversiones en REIT para poder invertir en estos instrumentos.

En la década de 1980 se vio un periodo de exceso de construcción en el que muchos desarrolladores inmobiliarios no lograron desplazar propiedades con un alto grado de apalancamiento y que no pudieron ser refinanciadas. Aunado a un incremento en el incumplimiento de los pagos de hipotecas, esto provocó el colapso de los precios de bienes raíces en la década de 1990. En consecuencia, estas empresas necesitaban fortalecer sus finanzas y buscar nuevas fuentes de capital. Por otro lado, las aseguradoras de riesgo clasificaron como riesgosas a la mayoría de las hipotecas comerciales de renta fija en 1993, por lo que fueron necesarias reservas de capital que descompensaron las finanzas de las aseguradoras. Esto hizo que la deuda de bienes raíces fuera una inversión poco atractiva para las compañías de seguros.

Los cambios de legislación dio a propietarios y administradores la capacidad de gestionar sus inmuebles. Esta disposición fue considerada como el "cambio más importante en impuestos del régimen de los REIT que ha permitido el crecimiento explosivo de la industria de REIT, para 
convertirlos en compañías operativas reales". Sin embargo, tomó unos años para que la legislación de 1986 ayudara a que la industria de los REIT experimentara un crecimiento explosivo (Brounen y Koning, 2014).

Después de estos cambios, los administradores de bienes raíces permitirían a los REIT reflejar las características de inversión de propiedades y crear ventajas de economía de escala y liquidez. El año 1998 fue desastroso para estos fideicomisos en los Estados Unidos: al parecer, sin ningún cambio en la valoración de las propiedades subyacentes, las primas de utilidad al valor neto de activos desaparecieron súbitamente en toda la industria. El índice NAREIT (National Association of Real Estate Investment Trusts) perdió $22 \%$ en ese año y en 1999 el descuento total del valor neto de activos fue de $18 \%$.

Con estos cambios preocuparon a los inversionistas respecto a los fundamentos de los REIT pues bien podrían sustituir sus inversiones con ofertas de otras acciones. El declive de la industria del REIT coincidió con la burbuja del punto com. La opinión generalizada era que los inversionistas sacarían su dinero del sector inmobiliario para invertirlo en las acciones tecnológicas, pues en teoría serían más rentables. Esta repentina tendencia del mercado de capitales pudo influir en el rendimiento de los REIT, con lo que se formuló una pregunta fundamental acerca de la lista de inversiones en bienes raíces: ¿las acciones de propiedades inmobiliarias son un reflejo de la propiedad o son una parte del mercado de valores? (Lee y Stevenson, 2005).

La caída del punto como proporcionó a los REIT nuevas oportunidades de acceso al capital después de un número de escándalos corporativos. A raíz de esto nació la Ley Sarbanes-Oxley de 2002, misma que hace cumplir estrictas disposiciones sobre la divulgación de información financiera de las sociedades con dinero público, lo que elevó de manera considerable el costo de salir a bolsa.

Por lo tanto, durante los primeros años de la década de 2000 aparecieron en su mayoría ofertas de ampliación de capital (SEO), pues los REIT ya buscaban inyecciones del mismo. Mientras tanto, el sector REIT maduró poco a poco y por lo tanto trató de librar los tiempos turbulentos.

La crisis inmobiliaria de 2008 puso en peligro a muchos REIT, ya que la deuda de bonos de titulización hipotecaria comercial y obligaciones de deuda garantizadas. El downturn inmobiliario resultó en una crisis económica mundial generalizada, lo que hizo que aumentara el costo de la deuda y se 
desplomaran los precios inmobiliarios, lo cual nuevamente puso a prueba a los REIT.

\section{Marco teórico}

Tras el éxito de la industria de REIT en EUA distintos países instituyeron estructuras inmobiliarias similares en un intento de facilitar el desarrollo de sus industrias nacionales de bienes raíces. En 1969, Holanda fue el primer país en aplicar la exención de impuestos de las empresas de bienes raíces, con lo que instituyó el Fiscale Beleggingsinstelling o FBI. Los holandeses tienen una larga historia de afinidad a los bienes raíces: sus grandes fondos de pensiones crearon una estructura legal beneficiosa para invertir en propiedades inmobiliarias. Esto hizo que los legisladores holandeses prestaran especial atención a los desarrollos colocados en los Estados Unidos (Brounen, D. y Koning, S. 2014).

En el Reino Unido, los UK-REIT se convirtieron en una realidad el 1 de enero de 2007 tras una disposición de la Ley de Hacienda de 2006. Inmediatamente, nueve empresas de bienes raíces fueron elegidas para convertirse en REIT y muchas más siguieron poco después. De esta manera, a finales de junio de 2009, el Reino Unido contaba con 21 REIT con una capitalización de mercado combinada de 23 mil millones de dólares.

Los UK-REIT fueron empresas que en un principio debían distribuir el $90 \%$ de sus beneficios con el fin de recibir los beneficios fiscales asociados a la estructura REIT. Sin embargo, a medida que otros países europeos pusieron en práctica estructuras REIT más accesibles, el Reino Unido anunció una reforma a su régimen en la materia: la Ley del Impuesto sobre Sociedades de 2010 proporcionó simplificaciones a las disposiciones relativas a UK-REIT. En UK se percibe que las ofertas inmobiliarias comerciales inversores un retorno de ingresos estable y relativamente alto, así como tener otros ventajas como su papel en la diversificación de la cartera (Investment Property Forum, 2005). En Baum y Devaney (2008) mencionan que este tipo de Mercado tiene la desventaja de la depreciación de las propiedades lo cual reduce los ingresos lo cual sería fuente de volatildad.

En Alemania, el 30 de marzo de 2007, se creó la Ley de Fideicomisos de Inversión Inmobiliaria, que permitió que las empresas establecieran un 
REIT. Al igual que EUA —su principal competidor-, los REIT no están sujetos a los impuestos de sociedades, pero son necesarios para distribuir la mayor parte de sus ingresos anuales en concepto de dividendos a los accionistas. Estos REIT tienen dos diferencias principales en comparación con los internacionales: la negativa es que no está permitida la incorporación de propiedad residencial construida antes del 1 de enero 2007; la positiva es que el impuesto sobre las ganancias de capital —que normalmente es de $40 \%$ en Alemania - se reduce a la mitad para la venta de propiedades de REIT, por lo que es una estructura muy atractiva para los compradores (Busching, 2007).

Ante un mercado inmobiliario angustiado, el gobierno español publicó la legislación para implementar Sociedades Cotizadas de Inversión en el Mercado Inmobiliario (SOCIMI). En España a diferencia de otros REIT europeos, las SOCIMI querían disfrutar de un estado totalmente exento de impuestos, pero están sujetas a un 18\% del impuesto de sociedades (Brounen, D. y Koning, S. 2014).

En Roig y Soriano (2015) mencionan que los REITs tienen una liquidez moderada en la actualidad aunque se prevé una mejora en el medio plazo y de series históricas de rentabilidades más amplias.

Australia fue uno de los primeros países en implementar una estructura de REIT, conocida en ese entonces como Listed Property Trusts (LPT), el primero de los cuales fue General Property Trusts (GPT), que comenzó a cotizar en la bolsa de valores en 1971. Australia también permite REIT privados, conocidos como fideicomisos de propiedad no cotizadas en público. Desde marzo de 2008, la legislación en la materia fue modificada y se crearon los fideicomisos llamados REIT australianos (A-REIT). El mercado en Australia es particularmente grande, en el 2009 contaba con una capitalización de mercado combinada de 43 mil millones dólares, que comprende $12.2 \%$ del mercado mundial de REIT, según la NARIT. La maduración del mercado REIT australiano ha permitido que estas entidades disfruten de un buen comienzo en la internacionalización de la industria, con lo que muchos A-REIT son ahora activos fuera de Australia.

Japón introdujo en el 2000 los J-REIT con la creación de fideicomisos de inversiones y la Ley de Sociedades de Inversión. En noviembre del año siguiente cotizaron los primeros dos J-REIT en la Bolsa de Tokio: el Fondo de Fortalecimiento de la Oficina del Japón y el Japan Real Estate Investment 
Corporation (Ooi J.T.L., Newell, G. y Sing, T.F., 2006). En marzo de 2009, Japón tenía 41 J-REIT con un valor de mercado de alrededor de 25 mil millones de dólares.

En América del Sur, varios países han puesto en práctica estructuras similares a los REIT. Puerto Rico (1972), Chile (1989), Brasil (1993) y Costa Rica (1994) fueron muy por delante de la explosión de los REIT en Asia y Europa. Los motivos para que estos países latinoamericanos adoptasen dichos regímenes fueron las mismas necesidades que en su momento tuvo el sector inmobiliario de EUA para el desarrollo de los REIT. Sin embargo, el tamaño de la industria en América del Sur es pequeño cuando se compara con Asia, América del Norte y Europa.

En México los REIT se denominan fideicomisos de infraestructura y bienes raíces (FIBRA). Nacieron en 2004 con un régimen fiscal, pero no fue sino hasta marzo de 2011 que apareció Fibra Uno como el primer REIT en nuestro país. La BMV tenía la visión de ofrecer un "supermercado financiero" en el que los inversionistas mexicanos tuvieran acceso a un amplio rango de productos de inversión como los FIBRA, utilizando su casa de bolsa local. En consecuencia, la primera oferta pública inicial (OPI) de un REIT tardó más de 6 años en que se realizara (BMV 2013).

En la medida en que la inversión extranjera es fundamental para México, cabe tener en cuenta que los inversionistas buscan riesgo bajo. Por ello, los FIBRA son un producto muy solicitado por muchas instituciones como fondos de pensiones, aseguradoras y afianzadoras. A fin de proporcionar la certeza que buscan los inversionistas, y las regulaciones dan más transparencia a las operaciones de inversión puesto que son supervisadas por el mercado público de capitales.

Los Criterios Generales de Política Económica para 2016 mencionan que $36.6 \%$ del financiamiento al sector privado, por medio de la emisión de deuda privada, corresponde a certificados bursátiles fiduciarios inmobiliarios emitidos por fideicomisos de infraestructura en bienes raíces.

En el siguiente apartado se mencionará la operatividad del FIBRA en el contexto mexicano. 


\section{Modelo de las operatividad de las FIBRAS en México: estructura y beneficios}

Con base en el artículo 223 de la LISR, los FIBRA son un tipo particular de fideicomiso en el que "el fideicomitente y el fideicomisario pueden beneficiarse de un estímulo fiscal con algunos requisitos, con la finalidad de fomentar la inversión inmobiliaria en México" (BMV, 2013).

La descripción de un fideicomiso se detalla en la Ley General de Títulos y Operaciones de Crédito (LGTOC) en su artículo 381, donde señala que: "En virtud del fideicomiso, el fideicomitente transmite a una institución fiduciaria la propiedad o la titularidad de uno o más bienes o derechos, según sea el caso, para ser destinados a fines lícitos y determinados, encomendando la realización de dichos fines a la propia institución fiduciaria" (Medina-Mora, 2007).

Las partes en un fideicomiso son: (i) el fideicomitente, quien constituye el fideicomiso mediante la aportación de bienes al mismo, (ii) el fiduciario, la institución autorizada que adquiere la titularidad de los bienes aportados y busca la consecución del fin, y (iii) el fideicomisario, quien recibe el provecho que el fideicomiso implica.

El fideicomitente. Es la persona física o moral que mediante una manifestación expresa de su voluntad, y teniendo la capacidad legal para ello, afecta la propiedad o titularidad de ciertos bienes o derechos a la institución fiduciaria para constituir un fideicomiso con el propósito de que se obtengan con ello los fines para los que éste se constituye.

La fiduciaria. Solo las instituciones expresamente autorizadas para desempeñarse como instituciones fiduciarias por la LIC tendrán tal carácter. Ahora bien, de conformidad con la LGTOC, el fiduciario (i) tendrá todos los derechos y acciones que se requieran para el cumplimiento del fideicomiso, (ii) estará obligado a cumplir dicho fideicomiso conforme al contrato de fideicomiso 0 acto constitutivo, (iii) no podrá excusarse o renunciar a su encargo, salvo por causas graves a juicio de un juez, y (iv) deberá responder por las pérdidas o menoscabos, daños y perjuicios que sufran los bienes fideicomitidos por negligencia grave de su parte.

El fideicomisario. Es la persona física o moral a la que se le otorga el beneficio del fideicomiso y la que recibe los remanentes una vez cumplida la finalidad del mismo. Puede ser fideicomisario toda persona física o moral que 
tenga la capacidad necesaria para salvaguardar el provecho que el fideicomiso implica.

Por otra parte, es necesario resaltar que, para obtener los beneficios que ofrece este estímulo de los FIBRA, se debe satisfacer una serie de requisitos, a saber:

1) El fideicomiso debe constituirse conforme a la legislación vigente en México.

2) La fiduciaria deberá ser una institución de crédito autorizada para actuar como tal y residente en México.

3) El fideicomiso debe tener una finalidad como adquisición o construcción de bienes inmuebles destinados al arrendamiento, adquisición del derecho a percibir ingresos provenientes del arrendamiento de dichos bienes, otorgar financiamientos para dichos fines con garantía real (hipotecaria) de los bienes arrendados y el patrimonio del fideicomiso (bienes, derechos y créditos) debe estar invertido de la siguiente manera al menos 70 por ciento en bienes inmuebles y el restante en valores gubernamentales 0 acciones de sociedades de inversión con instrumentos de deuda.

Además, los bienes inmuebles que se construyan o adquieran deben destinarse al arrendamiento y no enajenarse antes de cuatro años después de su construcción. Si esto no se cumple, el fideicomiso pierde el beneficio fiscal y la fiduciaria deberá emitir CPO por los bienes que integran el patrimonio del fideicomiso. Estos CPO deberán colocarse en México entre el gran público inversionista 0 bien, ser adquiridos por un grupo de inversionistas de por lo menos diez personas que no sean partes relacionadas entre sí. Ninguno de estos inversionistas podrá ser propietario en lo individual de más de $20 \%$ de la totalidad de los CPO emitidos. Por último, la fiduciaria deberá distribuir a los tenedores de los CPO, a más tardar el 15 de marzo, el 95\% del resultado fiscal inmediato anterior generado por los bienes integrantes del patrimonio del fideicomiso.

Una vez constituido el fideicomiso y puesto en marcha, el beneficio proviene del tratamiento fiscal que se le puede dar a los FIBRA en nuestro México, ya que partiendo del título segundo de la LISR, el fiduciario determinará el resultado fiscal del ejercicio, el cual se dividirá entre el número de CP emitidos. Por otra parte, no tendrá la obligación de realizar pagos provisionales de ISR, ni de pagar IMPAC de acuerdo con la LGTOC (art. 
381). Además, la fiduciaria deberá distribuir los resultados fiscales del ejercicio inmediato anterior, como se detalla con anterioridad (BMV, 2013).

Las ventajas de las FIBRAS en México se dividen en Fiscales, empresariales y contables. En primer lugar si un fideicomiso cumple con todos los requisitos plasmados en la Ley del Impuesto sobre la Renta (LISR), entonces tendrá los siguientes estímulos y beneficios fiscales establecidos en el artículo 224 (Castañares, 2010).

1. Estos fideicomisos no realizan pagos provisionales del ISR. Además, se encuentran exentos del pago del Impuesto al Activo por los bienes, derechos, créditos o valores que integren el patrimonio del FIBRA.

2. Los fondos de pensiones y jubilaciones extranjeros estarán exentos del pago del ISR que correspondería por los ingresos que reciban provenientes de los bienes, derechos, créditos y valores del patrimonio del FIBRA, así como de la ganancia de capital que obtengan por la enajenación de los certificados de participación ordinaria (CPO). Los fondos de pensiones y jubilaciones nacionales podrán invertir hasta 10\% de sus reservas en los CPO emitidos por los FIBRA.

3. Cuando los CPO del FIBRA se encuentren colocados entre el gran público inversionista, las personas físicas residentes en México y los residentes en el extranjero estarán exentos del pago del impuesto sobre la renta por ganancia de capital que obtengan de la enajenación de los CPO a través del mercado de valores.

4. Los propietarios de los inmuebles que al aportarlos al fideicomiso reciban CPO por el valor total o parcial de dichos bienes, podrán diferir el pago del impuesto sobre la renta hasta el momento en que enajenen dichos certificados 0 bien el fiduciario enajene los inmuebles aportados a terceras personas.

5. Cuando los inmuebles aportados al fideicomiso sean arrendados de inmediato a los fideicomitentes por el fiduciario, podrán diferir el pago del ISR causado por la ganancia, obtenida en la enajenación de los bienes inmuebles, hasta el momento en que termine el contrato de arrendamiento, siempre que éste no tenga un plazo mayor a diez años; 0 bien, hasta el momento en que el fiduciario enajene los bienes aportados.

En segundo lugar las ventajas desde una perspectiva empresarial el uso de las FIBRAS 1) fomenta la inversión inmobiliaria 2) fortalece la liquidez para desarrolladores 3) logra economías de escala 4) permite la 
capitalización de empresas inmobiliarias al aportar su inmueble al fideicomiso y 5) permite acceder a capitales más económicos que los instrumentos bancarios típicos y finalmente (Medina-Mora, 2007)

En tercer lugar, en lo relativo a la parte contable, cabe señalar que no se pagan impuestos de traslación de dominio, hay exenciones fiscales según la LISR, art. 223 y 224, y el impuesto al activo IMPAC es exento. Esto representa un beneficio atractivo para los inversionistas, pues no pagan impuestos por entrar, mantenerse ni salir de este tipo de inversiones. Sin dicho mecanismo, será gravoso este tipo de operaciones (Castañares, 2010). Las regulaciones que ofrecen los FIBRA mitigan riesgos, además de que las normas exigen un alto nivel de gobierno corporativo y una empresa profesional con experiencia en el ramo debe llevar la administración del inmueble. Medina-Mora, 2007)

\section{Análisis documental del mercado de las Fibras}

A través del análisis de contenido cualitativo (Andréu, 2013) buscamos explicar la popularidad de los REIT a nivel global haciendo un análisis de su evolución y crecimiento, sus similitudes y diferencias entre los países que se presentó en el marco teórico.

En la Tabla 1 se presenta un análisis de los principales riesgos y ventajas de los REITs. En donde se muestra que Holanda fue de los primeros países que instituyó los REITs. Además podemos destacar que este país tiene una estructura beneficiosa para las operaciones de estos instrumentos aunque los riesgos podrían ser la madurez del mercado. Por otra parte las ventajas de estímulos fiscales se presentan en países europeos como Reino Unido, Alemania y México. En relación a los riesgos de los REITs sería la depreciación de los inmuebles y la volatilidad que se genera en sus ingreso presentada en Reino Unido así como efectos fiscales en el caso de España.

Para el caso de México en donde se profundizó más a fondo en sus ventajas podríamos citar que derivado a las disposiciones legales de las FIBRAS están bajo una legislación rigurosa, lo cual mitiga los riesgos. Por otra parte, tiene muchas ventajas en la empresa ya que permite economías de escala, fomenta la inversión y el acceso a mercados de capitales. Por último favorece las exenciones fiscales. 
Tabla 1. Análisis comparativo de ventajas y riesgos de REITS

\begin{tabular}{|c|c|c|c|}
\hline País & $\begin{array}{l}\text { Año de operación } \\
\text { de REITS }\end{array}$ & Ventajas & Riesgos \\
\hline Holanda & 1969 & $\begin{array}{l}\text { Estructura legal beneficiosa y } \\
\text { grande }\end{array}$ & Maduro \\
\hline Reino Unido & 2007 & $\begin{array}{l}\text { Estímulos fiscales y } \\
\text { simplificaciones de } \\
\text { disposiciones de UK-REIT }\end{array}$ & $\begin{array}{l}\text { Depreciación de } \\
\text { inmuebles y } \\
\text { volatilidad en } \\
\text { ingresos }\end{array}$ \\
\hline Alemania & 2007 & $\begin{array}{l}\text { El impuesto sobre las } \\
\text { ganancias de capital se reduce } \\
\text { a la mitad para la venta de } \\
\text { propiedades de REIT }\end{array}$ & $\begin{array}{l}\text { No está permitida } \\
\text { la incorporación } \\
\text { de propiedad } \\
\text { residencial } \\
\text { construida antes } \\
\text { del } 1 \text { de enero } \\
2007\end{array}$ \\
\hline España & 2007 & Baja liquidez & $\begin{array}{l}18 \% \text { de } \\
\text { impuestos de } \\
\text { sociedades }\end{array}$ \\
\hline Australia & 1971 & $\begin{array}{l}\text { Mercado grande con presencia } \\
\text { internacional }\end{array}$ & Mercado maduro \\
\hline Japón & 2000 & Mercado en crecimiento & \\
\hline $\begin{array}{l}\text { América del } \\
\text { Sur }\end{array}$ & $\begin{array}{c}\text { Puerto Rico (1972), } \\
\text { Chile (1989), Brasil } \\
\text { (1993) y Costa Rica } \\
\text { (1994) }\end{array}$ & & $\begin{array}{l}\text { Pequeño en } \\
\text { comparación } \\
\text { Asia, América del } \\
\text { Norte y Europa. }\end{array}$ \\
\hline México & 2004 & $\begin{array}{l}\text { Mercado transparente, Ventajas } \\
\text { impositivas, fomento a la } \\
\text { inversión y la liquidez, acceso a } \\
\text { mercados de capitales más } \\
\text { económicos y mitigan riesgos }\end{array}$ & Crecimiento lento \\
\hline
\end{tabular}




\section{Conclusiones}

Como se observa, los REIT/FIBRA son un mecanismo de inversión que financian grandes propiedades inmobiliarias no sólo en América, sino en todo el mundo. Esto permite que el mercado de dinero público participe en estas inversiones, aunque en su historia han presentado diferentes riesgos y rendimientos debido al marco legal de sus países de origen.

Entre los principales riesgos de este mercado son la depreciación de los bienes inmueble ( Baum y Devaney, 2008), la no incorporación de la propiedad residencial y en algunos países Latinoamericanos su tamaño pequeño en comparación a fondos Europeos o de Estados Unidos. Sus principales ventajas son los estímulos fiscales, la inversión que generan, el acceso a mercados de capitales, entre otros.

En México, la estructura legal de los FIBRA es muy robusta y parecida a la de EUA. Uno de los motores de estos instrumentos es la exención fiscal que ofrecen. Además se ha dado un gran crecimiento en países europeos como el caso de Reino Unido contrario de lo encontrado en España siguen sin motivar el interés del mercado en la medida en que no ofrece estos beneficios fiscales.

Existe una gran atención en todo el mundo por estos instrumentos. La gran pregunta será hasta qué punto son opciones viables para usarlos como mecanismo de diversificación a fin de disminuir el riesgo o aumentar el rendimiento.

\section{Referencias}

Andréu Abela, J. (2000). Las técnicas de análisis de contenido: una revisión actualizada. Sevilla: Fundación Centro de Estudios Andaluces.

Baum, A. \& Devaney, S. (2008). Depreciation, income distribution and the UK REIT, Journal of Property Investment \& Finance, 26(3), 195-209.

BMV (2013). Bolsa Mexicana de Valores. [En línea] http://www.bmv.com.mx/ [consultado $17 /$ nov/2016].

Brounen, D. \& Koning, S. (2014). 50 years of real estate investment trusts: an international examination of the rise and performance of REIT, Journal of Real Estate Literature, 20(2), 197-230.

Busching, T. (2007). Germany enters the REIT universe with a big bang, Journal of Retail and Leisure Property, 6(3), 181-187. 
Castañares, J. A. (2010). Fideicomisos de infraestructura y bienes raíces. ¿Alternativa real para el financiamiento de ciudades-marcas?. [En línea] http://www.direccionestrategica.itam.mx/wp-content/uploads/2010/06/FIBRASfinal.doc [consultado 17/nov/2016].

Decker, M. (1998). The modern real estate investment trust industry: an overview. En Garrigan, R. \& Parsons, J. (eds.), Real estate investment trusts: structure, analysis, and strategy. Nueva York: McGraw-Hill, pp. 3-8.

Graff, R. (2001). Economic analysis suggests that REIT investment characteristics are not as advertised, Journal of Real Estate Portfolio Management, 7(2), 99-124.

Gyourko, J. \& Keim, D. (1992). What does the stock market tell us about real estate returns? Real Estate Economics, 20(3), 457-85.

Investment Property Forum (2005). Understanding commercial property investment: A guide for financial advisers. London: Investment Property Forum.

Roig J. \& Soriano J. (2015). "Revista Europea de Dirección y Economía de la Empresa" 24, 92-107

Lee, S. \& Stevenson, S. (2005). The case for REITs in mixed-asset portfolio in the short and long run, Journal of Real Estate Portfolio Management, 11(1), 55:80.

Medina-Mora, I. (2007). Fideicomisos de Infraestructura y Bienes Raíces. Tesis de licenciatura. México: Instituto Tecnológico Autónomo de México.

Parsons, J. (1998). REITs and institutional investors. En Garrigan, R. y Parsons, J. (eds.), Real estate investment trusts: structure, analysis, and strategy. Nueva York: McGrawHill.

Ooi, J.T.L., Newell, G. \& Sing, T.F. (2006). The growth of REIT markets in Asia. Journal of Real Estate Literature, 14(2), 204-222

SHCP (2015). Criterios generales de política económica, Secretaria de Hacienda y Crédito Público. [En línea] http://www.ppef.hacienda.gob.mx/work/models/PPEF/2016/docs/ paquete/CGPE_2016.pdf [consultado 15/nov/2015].

Souza, L. (2014). Modern real estate portfolio management (MREPM): Applications in modern and post-modern real estate portfolio theory (MREPT/PMREPT). Dissertetion thesis to obtain the degree of Doctorate of Philosophy in Business Administration (DBA) with a concentration in Corporate Finance, San Francisco: Golden Gate University, Edward S. Ageno School of Business. 